\title{
Marketing y Responsabilidad Social Empresarial \\ Un mapa de navegación
}

DOI: $10.22403 /$ UQROOMX/TYP04/08

חוח

Alejandro Alvarado Herrera*

Resumen

El resurgimiento del interés de empresarios, profesionales y académicos por la Responsabilidad Social Empresarial (RSE) ha propiciado tal abundancia de aproximaciones teóricas interdisciplinarias, que confunde e interfiere en la selección de las más pertinentes. Para los especialistas en marketing, la dificultad es aún mayor, pues los esquemas de identificación y categorización de dichas teorías provienen de otros campos del saber, y los escasos modelos del marketing presentan vacíos de conocimiento. El presente trabajo ofrece una panorámica general de las perspectivas teóricas sobre la RSE, su naturaleza y su aplicación en el ámbito del marketing. La propuesta forma parte de una investigación desarrollada a partir de la exploración exhaustiva de I I 35 artículos científicos publicados en los últimos treinta y cinco años en revistas líderes indexadas, así como de la integración de varios de los esquemas de clasificación. Las conclusiones señalan posibles líneas de investigación futuras.

Palabras $\mid$ Responsabilidad Social Empresarial, estado-de-la-cuestión, CLAVE marketing, revisión de la literatura, teorías.

*Universidad de Quintana Roo/alealva@uqroo.mx/alhe@alumni.uv.es 


\section{Introducción}

La Responsabilidad Social Empresarial (RSE)* -o Corporativa (RSC) (Bigné, Chumpitaz,Andreu y Swaen, 2005)- ha sido formalmente estudiada desde antes de la II Guerra Mundial (van Oosterhout y Heugens, 2006)' y ha motivado un amplio debate en la literatura de negocios (Carroll, 1999; Dacin y Brown, 2006; Daub y Ergenzinger, 2005; Ellen, Webb y Mohr, 2006; Maignan y Ferrell, 2004; Polonsky, 2005), al menos desde la publicación del libro Social Responsibilities of the Businessmen, de Howard Bowen en 1953 (Carroll, 1999).

La revisión y redefinición constante de los principios de la RSE (Balmer, 200 I; Lockett, Moon y Visser, 2006; Wartick y Cochran, 1985), determinada por su propio carácter controvertido (Carrigan y Attalla, 200 I), ha propiciado la generación y la aplicación de una multitud de perspectivas teóricas y terminológicas (Garriga y Melé, 2004) que suscitan confusión (Lantos, 200I) a tal grado, que el desarrollo de este campo de conocimiento ha sido descrito en términos kuhnianos como en un "continuo estado de emergencia" (Lockett et al., 2006: I33), e incluso se ha propuesto abandonar su estudio en favor de marcos conceptuales "más poderosos y mejor establecidos" (van Oosterhout y Heugens, 2006: 3).

No obstante, tal dimisión parece estar lejos de suceder, pues como apunta Ballesteros (2004:4) “filantropía, marketing con causa, acción social de la empresa, responsabilidad social corporativa, etc. son términos novedosos para el mercado y atractivos para los que tenemos la responsabilidad de pensar y aportar consideraciones sobre ello" con lo cual y visto el renovado interés de empresas, profesionales y académicos por la RSE (Maignan y Ralston, 2002; McWilliams, Siegel yWright, 2006), queda por delante "un largo camino que recorrer" (Fernández, 2004: 36) y una complejidad aún mayor parece estar garantizada.

Este escenario ha motivado la necesidad de aminorar la desorientación referida, para lo cual han surgido investigaciones que buscan organizar las teorías

*Este trabajo es un extracto de la investigación ganadora del tercer lugar nacional del XI Premio Junior AEDEMo 2006, auspiciado por la Asociación Española de Estudios de Mercado, Marketing y Opinión.

'De acuerdo con van Oosterhout y Heugens (2006: 6): "la dimensión social de las actividades económicas ya estaba en la agenda académica” con contribuciones como las de Clark (1919) y Berle (1931). 
relacionadas con la RSE en función de distintos criterios, tales como: su evolución temporal (Carroll, 1999; Murphy, I978, Wood, I99Ib), su enfoque (Windsor, 2006), la evolución de sus contenidos (De Bakker, Groenewegen y Den Hond, 2005 y 2006; Gerde y Wokutch, 1998), el rol de los negocios en la sociedad (Garriga y Melé, 2004; Zenisek, 1979), su naturaleza teórica (McWilliams et al., 2006) y su aplicabilidad (Nasi, Nasi, Phillips y Zyglidopoulos, 1997).

Sin embargo, $y$ a pesar de la preeminencia del marketing en las actividades estratégicas de RSE (Lantos, 200 I; Marín, 2004), la mayor parte de esos trabajos de categorización provienen del ámbito más amplio del management, siendo escasos los originados en el marketing donde, en concreto, se ha propuesto clasificar dichas perspectivas teóricas en función del rol de los negocios en la sociedad (Lantos, 200I) y se han identificado las principales unidades de análisis y dimensiones de la RSE consideradas por los investigadores de marketing (Maignan y Ferrell, 2004). Estos incipientes esfuerzos demandan ser complementados, explorando en profundidad las principales perspectivas teóricas con las que ha sido estudiada la RSE, su naturaleza y los aspectos que han sido abordados por el marketing.

\section{Objetivo y metodología del trabajo}

El presente trabajo tiene como objetivo aportar elementos para exponer una panorámica general de las perspectivas teóricas sobre la RSE, su naturaleza y su aplicación en el ámbito del marketing, mediante la creación de un "mapa de navegación" capaz de responder a las siguientes preguntas:

I) ¿Cuáles son las principales perspectivas teóricas con las que ha sido estudiada la RSE y cuál es su naturaleza?, y

2) ¿Qué aspectos de la RSE han sido abordados por el marketing con las teorías en cuestión?

\section{Metodología}

Se diseñó una investigación exploratoria basada en una exhaustiva revisión cualitativa de la literatura de RSE que permitiera identificar de una manera válida y confiable las contribuciones esenciales sobre el tema.

Recientemente, investigadores holandeses de la Vrije Universiteit publicaron el método que emplearon para reunir, extensivamente, artículos acerca 
de la RSC. Este método sirvió de modelo para la conformación de la base de datos que fundamenta esta investigación. El procedimiento consiste en realizar búsquedas de términos específicos relacionados con la RSE en los campos título, palabras claves y resumen de los índices especializados: ABI/Inform y WoS/sSCl, y en su posterior depuración manual, atendiendo a criterios de no duplicidad y de pertenencia a los ámbitos de estudio de management y de marketing (los términos y criterios puntuales pueden consultarse en De Bakker et al. 2005 y 2006).

De Bakker y sus colaboradores crearon una base de datos con los trabajos publicados entre los años 1972 y 2002, la cual compartieron con el autor de este estudio para su actualización mediante la metodología descrita, a fin de garantizar la integridad del contenido resultante. El 4 de octubre de 2006 se hizo la última actualización y depuración para este trabajo y se obtuvieron II 35 artículos de revistas científicas indizadas, que fueron analizados para identificar las contribuciones representativas, claves o seminales en la propia literatura y hacer su caracterización.

Después, a través de la integración y aplicación de tres de las categorizaciones, se esbozó un panorama general de las teorías usadas en la RSE; fue elaborada la propuesta para su clasificación y la administración específica de los trabajos que abordan el tema desde el marketing.

\section{Análisis e integración}

\section{Perspectivas teóricas de la RSE y su naturaleza}

Para conocer las principales perspectivas teóricas de la RSE y a su naturaleza se definieron tres clasificaciones, para lo cual resultó especialmente adecuado el trabajo de Garriga y Melé (2004), ya que engloba las teorías más relevantes sobre el tema y establece cuatro categorías: teorías instrumentales, teorías políticas, teorías integradoras y teorías éticas, de las que se desprenden 14 subcategorías que permiten clasificar otras aportaciones.

No obstante sus ventajas, el esquema de estos autores requería ser complementado debido a que: a) no permite identificar el tipo de trabajo de las contribuciones; b) no contempla expresamente la naturaleza de las teorías clasificadas, y c) al centrarse en el ámbito del management, no indaga en profundidad sobre los trabajos realizados por los investigadores de marketing. Para subsanar estas lagunas, respectivamente se emplearon los trabajos de De Bakker et al. (2005), McWilliams et al. (2006) y Maignan y Ferrell (2004). 
El trabajo de De Bakker et al. (2005) nos proporcionó la variable el tipo de trabajo, que se refiere a la orientación epistemológica de los artículos, la cual puede ser conceptual, exploratoria, predictiva, instrumental, normativa o descriptiva, según lo expuesto en el cuadro $I$.

Cuadro I. Posibles orientaciones epistemológicas de los trabajos DE INVESTIGACIÓN (TIPOS DE TRABAJOS)

\begin{tabular}{|c|c|c|}
\hline \multirow[t]{3}{*}{ Teóricos } & Conceptual & $\begin{array}{l}\text { Su principal enfoque está en el desarrollo } \\
\text { de proposiciones, hipótesis o correlaciones } \\
\text { entre constructos teóricos basándose en } \\
\text { una discusión del estado-de-la-cuestión de } \\
\text { la literatura; no ha sido recolectado nuevo } \\
\text { material empírico para este trabajo. }\end{array}$ \\
\hline & Exploratorio & $\begin{array}{l}\text { Su principal enfoque está en el desarrollo de } \\
\text { proposiciones, hipótesis y correlaciones entre } \\
\text { constructos teóricos y se basa en el estudio } \\
\text { de nuevos y extensivos datos empíricos. }\end{array}$ \\
\hline & Predictivo & $\begin{array}{l}\text { Su principal enfoque está en testar (refutar, con- } \\
\text { firmar) proposiciones, hipótesis o correlaciones } \\
\text { entre constructos teóricos y se basa en el } \\
\text { estudio de nuevos y extensivos datos empíricos. }\end{array}$ \\
\hline \multirow[t]{2}{*}{ Prescriptivos } & Instrumental & $\begin{array}{l}\text { Su principal enfoque está en proveer pres- } \\
\text { cripciones (medios, ideas, recetas para accio- } \\
\text { nar) a practicantes y profesionales, que sean } \\
\text { instrumentos para la realización de algún fin } \\
\text { deseado, tal como mejorar el desempeño de } \\
\text { alguna dimensión. }\end{array}$ \\
\hline & Normativo & $\begin{array}{l}\text { Su principal enfoque está en proveer } \\
\text { prescripciones (medios, ideas, recetas para } \\
\text { accionar) a practicantes y profesionales, } \\
\text { que sean valiosas en sí mismas cuando se } \\
\text { consideran desde algún punto de vista ético, } \\
\text { moral o religioso. }\end{array}$ \\
\hline Descriptivos & Descriptivos & $\begin{array}{l}\text { Su mayor enfoque está en el reporte de } \\
\text { hechos u opiniones; no tiene intención de } \\
\text { contribuir de forma teórica ni prescriptiva. }\end{array}$ \\
\hline
\end{tabular}

Fuente: Adaptado de De Bakker, Groenewegen y Den Hond (2005). 
Marketing y Responsabilidad

Social Empresarial

Del trabajo de McWilliams et al. (2006) obtuvimos la variable naturaleza teórica de las contribuciones estudiadas; las teorías específicas se señalarán en los cuadros correspondientes. En cuanto a la forma de aprovechamiento de la investigación de Maignan y Ferrell (2004), ésta se discute en el apartado "La RSE en el marketing”. La figura I muestra la lógica de la clasificación y sirve de guía para la comprensión de la estructura de este epígrafe.

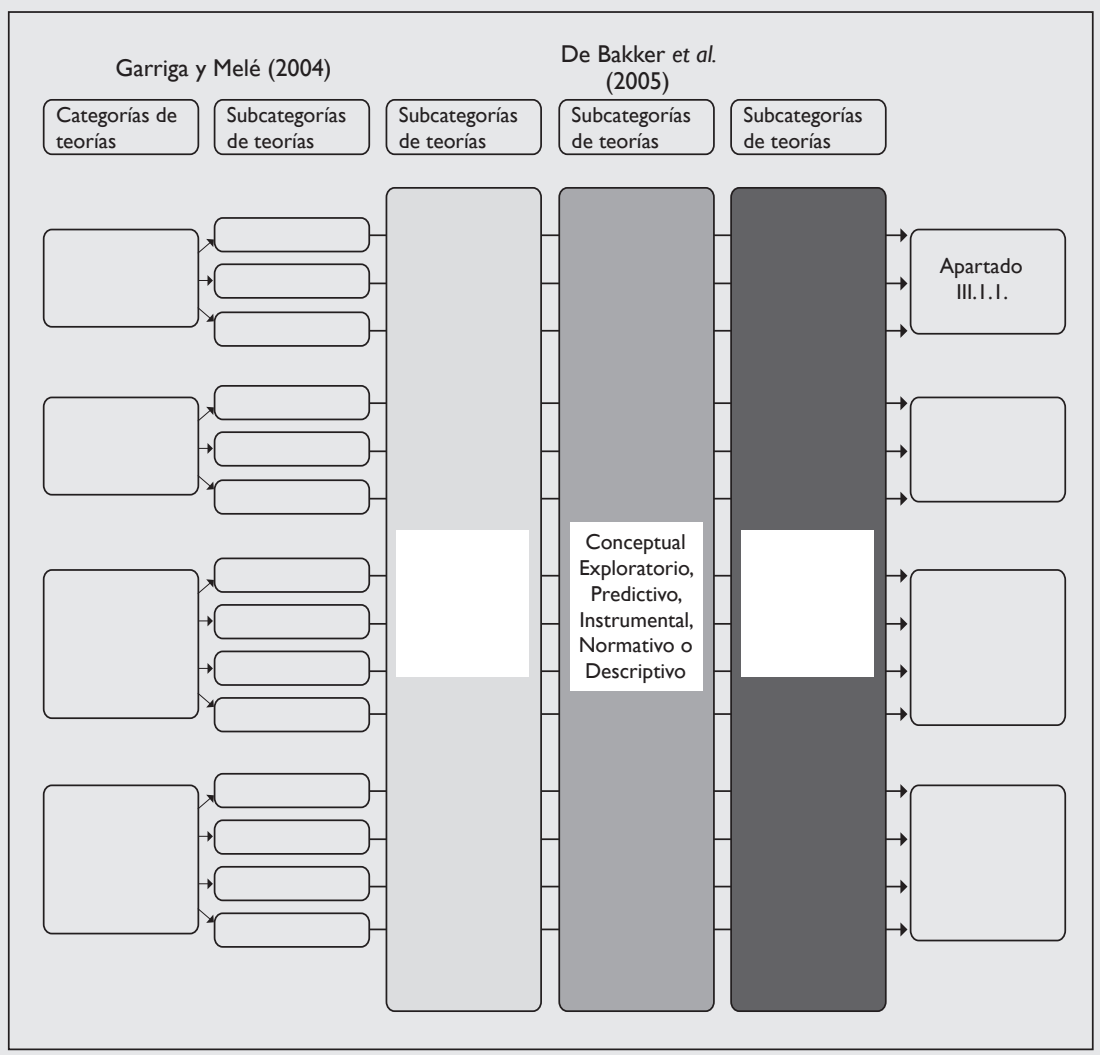

Fuente: Elaboración propia.

Figura I. Lógica de integración de las clasificaciones seleccionadas 


\section{Categorías descritas por Garriga y Melé}

I) Teorías instrumentales

Agrupa las contribuciones que conciben a la RSE como "una herramienta estratégica para alcanzar los objetivos económicos y, en última instancia, la creación de riqueza" (2004:53) y consta de tres subcategorías:a) Maximización del valor de los accionistas (shareholders); b) Estrategias para lograr ventajas competitivas, y c) Marketing relacionado a alguna causa. Según Garriga y Melé, todas las teorías de este grupo mantienen un enfoque hacia el cumplimiento de los objetivos económicos a través de actividades sociales.

De las 25 teorías incorporadas en el cuadro 2, llama la atención el equilibrio en la orientación epistemológica de los trabajos pertenecientes a la subcategoría de Marketing con causa, mientras que las consideradas en la subcategoría de la Maximización del valor de los accionistas son conceptuales.

Cuadro 2. Categoría de las teorías instrumentales

\begin{tabular}{|c|c|c|c|}
\hline $\begin{array}{l}\text { Naturaleza de la } \\
\text { perspectiva teórica }\end{array}$ & Contribuciones clave & Método & Tipo de trabajo \\
\hline & \multicolumn{3}{|c|}{ Subcategoría: Maximización del valor de los accionistas [shareholders] } \\
\hline \multirow[t]{5}{*}{$\begin{array}{l}\text { Teoría de la agencia } \\
\text { [Agency theory] }\end{array}$} & $\begin{array}{l}\text { Coelho, McClure y Spry } \\
\text { (2003a y 2003b) }\end{array}$ & Crítica académica & Conceptual \\
\hline & Friedman (1962) & Inductivo & Conceptual \\
\hline & Friedman (1970) & Inductivo & Conceptual \\
\hline & Friedman (1978) & Manifiesto & Conceptual \\
\hline & Jensen (2000) & Inductivo & Conceptual \\
\hline \multirow{3}{*}{$\begin{array}{l}\text { Teoría de la agencia } \\
\text { / Relativismo ético* }\end{array}$} & Bhide y Stevenson (1990) & Inductivo & Conceptual \\
\hline & Carr (1968) & Inductivo & Conceptual \\
\hline & Fieser (1996) & Inductivo & Conceptual \\
\hline
\end{tabular}


Marketing y Responsabilidad

Social Empresarial

Cuadro 2. Categoría de las teorías instrumentales

(Continuación)

\begin{tabular}{|c|c|c|c|}
\hline $\begin{array}{l}\text { Naturaleza de la } \\
\text { perspectiva teórica }\end{array}$ & Contribuciones clave & Método & Tipo de trabajo \\
\hline & \multicolumn{3}{|c|}{ Subcategoría: Estrategias para lograr ventajas competitivas } \\
\hline & $\begin{array}{l}\text { Hart y Christensen } \\
\text { (2002) }\end{array}$ & Inductivo & Instrumental \\
\hline & Porter y Kramer (2002) & Estudio de caso & Instrumental \\
\hline & $\begin{array}{l}\text { Prahalad y Hammond } \\
(2002)\end{array}$ & Inductivo & Instrumental \\
\hline \multirow{4}{*}{$\begin{array}{l}\text { Visión de los recur- } \\
\text { sos y las capacida- } \\
\text { des [Resource-based } \\
\text { view of the firm] }\end{array}$} & Hart (1995) & Inductivo & Conceptual \\
\hline & Litz (1996) & Inductivo & Conceptual \\
\hline & McWilliams y Siegel (200I) & Inductivo & Conceptual \\
\hline & $\begin{array}{l}\text { McWilliams, van Fleet } \\
\text { y Cory }(2002)\end{array}$ & Inductivo & Conceptual \\
\hline \multirow{2}{*}{$\begin{array}{l}\text { Teoría del lide- } \\
\text { razgo estratégico } \\
\text { [Strategic leadership } \\
\text { theory] }\end{array}$} & $\begin{array}{l}\text { Waldman, Siegel y } \\
\text { Javidan (2006) }\end{array}$ & $\begin{array}{l}\text { Hipotético- } \\
\text { deductivo }\end{array}$ & Predictivo \\
\hline & \multicolumn{3}{|c|}{ Subcategoría: Marketing relacionado a alguna causa } \\
\hline \multirow[t]{8}{*}{$\begin{array}{l}\text { Visión estratégica } \\
\text { de la firma }\end{array}$} & $\begin{array}{l}\text { Barone, Miyazaki y } \\
\text { Taylor (2000) }\end{array}$ & Hipotético-deductivo & Predictivo \\
\hline & Hoeffler y Keller (2002) & Inductivo & Conceptual \\
\hline & Lafferty y Goldsmith (2005) & Hipotético-deductivo & Predictivo \\
\hline & Murray y Montanari (1986) & Inductivo & Conceptual \\
\hline & Sen y Bhattacharya (200I) & Hipotético-deductivo & Predictivo \\
\hline & $\begin{array}{l}\text { Sen, Bhattacharya y } \\
\text { Korschun (2006) }\end{array}$ & $\begin{array}{l}\text { Hipotético- } \\
\text { deductivo }\end{array}$ & Predictivo \\
\hline & Varadarajan y Menon (1988) & Inductivo & Conceptual \\
\hline & Webb y Mohr (1998) & Hipotético-deductivo & Exploratorio \\
\hline
\end{tabular}

* El relativismo ético no fue considerado por McWilliams et al. (2006) por lo que la naturaleza teórica fue propuesta por el autor de este trabajo al estar implícita en los trabajos aquí referidos. Fuente: Elaboración propia. 
Cuadro 3. Categoría de las teorías políticas

\begin{tabular}{|c|c|c|c|}
\hline $\begin{array}{l}\text { Naturaleza de la } \\
\text { perspectiva teórica }\end{array}$ & Contribuciones clave & Método & Tipo de trabajo \\
\hline & & \multicolumn{2}{|c|}{ Subcategoría: Constitucionalismo corporativo } \\
\hline \multirow[t]{4}{*}{$\begin{array}{l}\text { Teoría del servidor } \\
\text { [Stewardship theory] }\end{array}$} & $\begin{array}{l}\text { Caldwell y Karri } \\
(2005)\end{array}$ & Inductivo & Conceptual \\
\hline & Davis (1967) & Inductivo & Conceptual \\
\hline & $\begin{array}{l}\text { Donaldson y Davis } \\
\text { (1991) }\end{array}$ & Hipotético-deductivo & Predictivo \\
\hline & \multicolumn{3}{|c|}{ Subcategoría:Teoría integradora del contrato social } \\
\hline \multirow{5}{*}{$\begin{array}{l}\text { Teoría integradora } \\
\text { del contrato social* } \\
\text { [Integrative social } \\
\text { contract theory] }\end{array}$} & $\begin{array}{l}\text { Donaldson y Dunfee } \\
\text { (1994) }\end{array}$ & Inductivo & Conceptual \\
\hline & $\begin{array}{l}\text { Donaldson y Dunfee } \\
\text { (1999) }\end{array}$ & Inductivo & Conceptual \\
\hline & $\begin{array}{l}\text { Dunfee, Smith y Ross } \\
\text { (1999) }\end{array}$ & Inductivo & Conceptual \\
\hline & Malan (2005) & Inductivo & Instrumental \\
\hline & $\begin{array}{l}\text { Watson, Shepard y } \\
\text { Stephens (1999) }\end{array}$ & Hipotético-deductivo & Predictivo \\
\hline
\end{tabular}

\begin{tabular}{llll}
$\begin{array}{l}\text { Ciudadanía } \\
\text { corporativa* [Corpo- } \\
\text { rate citizenship] }\end{array}$ & $\begin{array}{l}\text { Fombrun, Gardberg y } \\
\text { Barnett (2000) }\end{array}$ & Inductivo & Conceptual \\
& $\begin{array}{l}\text { Maignan y Ferrell } \\
(2000)\end{array}$ & Hipotético-deductivo & Predictivo \\
& $\begin{array}{l}\text { Maignan, Ferrell y } \\
\text { Hult (1999) }\end{array}$ & Hipotético-deductivo & Predictivo \\
& $\begin{array}{l}\text { Wood y Logsdon } \\
(2002)\end{array}$ & $\begin{array}{l}\text { Discurso } \\
\text { argumentativo }\end{array}$ & Conceptual \\
\hline
\end{tabular}

* Teoría no considerada por McWilliams et al. (2006) pero cuya naturaleza fue explicitada por los autores referidos en el cuadro.

Fuente: Elaboración propia. 


\section{2) Teorías políticas}

Consta de tres subcategorías: a) Constitucionalismo corporativo, b) Teoría integradora del contrato social, y c) Ciudadanía corporativa. Todas comparten su enfoque "en las interacciones y conexiones entre las empresas y la sociedad, y en el poder y la posición de las empresas y su inherente responsabilidad" (Garriga y Melé, 2004:55). Doce contribuciones fueron clasificadas aquí y como se aprecia en el cuadro 3 los trabajos de los investigadores de marketing han tendido a agruparse en la subcategoría Ciudadanía corporativa, lo que sugiere cierta desatención respecto al uso de las teorías del servidor y la integradora del contrato social en nuestra disciplina.

\section{3) Teorías integradoras}

Las teorías integradoras, aquellas que "buscan cómo integrar en las empresas las demandas sociales, argumentando que los negocios dependen de la sociedad para su existencia" (Garriga y Melé, 2004: 57), constituyen el siguiente espacio de clasificación, y Garriga y Melé distinguieron cuatro subcategorías:a) Aspectos administrativos o de gestión, b) Principio de responsabilidad pública, c) Gestión de grupos de interés [stakeholders], y d) Desempeño social corporativo. El cuadro 4 presenta las $2 \mathrm{I}$ contribuciones clasificadas en esta categoría.

Cuadro 4. Categoría de las teorías integradoras

\begin{tabular}{llll}
\hline $\begin{array}{l}\text { Naturaleza de la } \\
\text { perspectiva teórica }\end{array}$ & $\begin{array}{c}\text { Contribuciones } \\
\text { clave }\end{array}$ & Método & Tipo de trabajo \\
\hline $\begin{array}{l}\text { Teoría institucional [Insti- } \\
\text { tutional theory] /Respuesta } \\
\text { social corporativa* }\end{array}$ & $\begin{array}{l}\text { Ackerman } \\
(1973)\end{array}$ & Inductivo & Instrumental \\
[Corporate social response] & & & \\
$\begin{array}{l}\text { Teoría de los grupos } \\
\text { de interés [Stakeholder } \\
\text { theory] / Relativismo }\end{array}$ & $\begin{array}{l}\text { Blowfield } \\
\text { ético** }\end{array}$ & Estudio de caso & Instrumental \\
\hline
\end{tabular}


Cuadro 4. Categoría de las teorías integradoras

(Continuación)

\begin{tabular}{|c|c|c|c|}
\hline Naturaleza de la perspectiva teórica & $\begin{array}{l}\text { Contribuciones } \\
\text { clave }\end{array}$ & Método & $\begin{array}{l}\text { Tipo de } \\
\text { trabajo }\end{array}$ \\
\hline \multicolumn{4}{|c|}{ Subcategoría:Aspectos administrativos o de gestión } \\
\hline $\begin{array}{l}\text { Teoría institucional [Institutional } \\
\text { theory] /Respuesta social corporati- } \\
\text { va* [corporate social response] }\end{array}$ & Ackerman (1973) & Inductivo & Instrumental \\
\hline $\begin{array}{l}\text { Teoría de los grupos de interés } \\
\text { [Stakeholder theory] / Relativismo } \\
\text { ético** }\end{array}$ & Blowfield (2004) & $\begin{array}{l}\text { Estudio de } \\
\text { caso }\end{array}$ & Instrumental \\
\hline $\begin{array}{l}\text { Teoría de los grupos de interés } \\
\text { /Respuesta social corporativa* /Teo- } \\
\text { ría institucional / Ética aplicada a la } \\
\text { empresa* [Moral/ethical theory] }\end{array}$ & Epstein ( 1987$)$ & Inductivo & Conceptual \\
\hline Teoría institucional & Jones (1980) & Inductivo & Conceptual \\
\hline $\begin{array}{l}\text { Teoría de los grupos de interés } \\
\text { /Teoría integradora del contrato } \\
\text { social* /Teoría institucional /Teoría } \\
\text { del desempeño social corporativo* } \\
\text { [Corporate social performance] }\end{array}$ & Moir (200I) & Inductivo & Conceptual \\
\hline $\begin{array}{l}\text { Visión estratégica de la firma /Teoría } \\
\text { de la respuesta social corporativa* }\end{array}$ & Sethi (1975) & Inductivo & Conceptual \\
\hline \multirow[t]{2}{*}{$\begin{array}{l}\text { Visión estratégica de la firma /Teoría } \\
\text { institucional }\end{array}$} & $\begin{array}{l}\text { Wartick y Mahon } \\
\text { (1994) }\end{array}$ & Inductivo & Conceptual \\
\hline & \multicolumn{3}{|c|}{ Subcategoría: Principio de responsabilidad pública } \\
\hline $\begin{array}{l}\text { Teoría de los grupos de interés } \\
\text { /Teoría de la agencia / Visión de los } \\
\text { recursos y las capacidades /Principio } \\
\text { de responsabilidad pública* [Public } \\
\text { responsibility principle] }\end{array}$ & Lea (1999) & $\begin{array}{l}\text { Estudio de } \\
\text { caso }\end{array}$ & Conceptual \\
\hline $\begin{array}{l}\text { Principio de responsabilidad públi- } \\
\text { ca*/Respuesta social corporativa* } \\
\text { /Teoría institucional }\end{array}$ & $\begin{array}{l}\text { Preston y Post } \\
\text { (1981) }\end{array}$ & Inductivo & Conceptual \\
\hline
\end{tabular}


Marketing y Responsabilidad

Social Empresarial

Cuadro 4. Categoría de las teorías integradoras

(Continuación)

\begin{tabular}{lccc}
\hline Naturaleza de la perspectiva teórica & $\begin{array}{c}\text { Contribuciones } \\
\text { clave }\end{array}$ & Método & $\begin{array}{l}\text { Tipo de } \\
\text { trabajo }\end{array}$ \\
\hline
\end{tabular}

Subcategoría: Gestión de grupos de interés [stakeholders]

Teoría de los grupos de interés /Teoría de la agencia / Teoría del servidor

Agle, Mitchell y

Teoría de los grupos de interés /

Visión de los recursos y las capacidades / Teoría de la firma / Teoría de la propiedad* [Property rights]

Teoría de los grupos de interés / Teoría de la agencia / Visión estratégica de la firma / Teoría del contrato social* / Ética aplicada a la empresa*

Teoría de los grupos de interés /

Teoría de la firma

Teoría de los grupos de interés/Teo- Rowley (1997) ría de la respuesta organizacional*

[Organizational responses theory] /

Visión estratégica de la firma
Sonnenfeld (1999)

Mitchell, Agle y

Wood (1997)

Hipotético- Predictivo

deductivo

Inductivo

Exploratorio

Asher, Mahoney y Inductivo

Conceptual

Mahoney (2005)

Jones (1995)

Inductivo

Exploratorio

Kochan y

Rubinstein (2000)

Estudio de

Exploratorio

caso

Inductivo

Exploratorio

Subcategoría: Desempeño social corporativo

\begin{tabular}{llll}
\hline $\begin{array}{l}\text { Teoría del desempeño social corpo- } \\
\text { rativo*Visión estratégica de la firma } \\
\text { / Teoría de la propiedad* }\end{array}$ & Baron (200I) & $\begin{array}{l}\text { Hipotético- } \\
\text { deductivo }\end{array}$ & Exploratorio \\
$\begin{array}{l}\text { Teoría del desempeño social } \\
\text { corporativo* / Respuesta social } \\
\begin{array}{l}\text { corporativa* / Ética aplicada a la } \\
\text { empresa* / Teoría del liderazgo } \\
\text { estratégico }\end{array}\end{array}$ & Carroll (1979) & Inductivo & Conceptual \\
\hline
\end{tabular}


Cuadro 4. Categoría de las teorías integradoras

(Continuación)

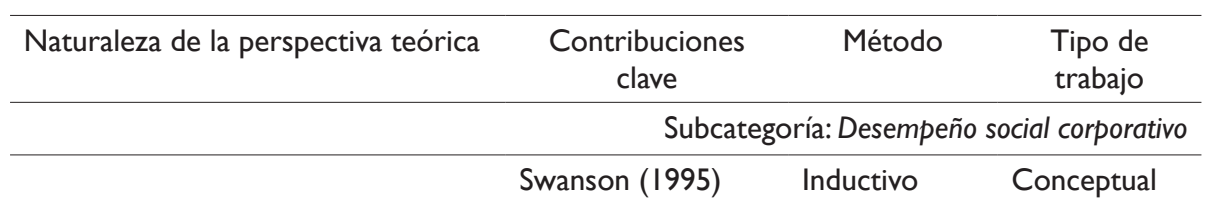

Swanson (1999) Inductivo Conceptual

Teoría del desempeño social corporativo* [corporate social performance] / Respuesta social corporativa* /Relativismo ético*/Teoría del servidor

Teoría del desempeño social corporativo* / Teoría de la agencia / Respuesta social corporativa*/Principio de responsabilidad pública*

Teoría del desempeño social corporativo* / Respuesta social corporativa*

Wartick y Cochran Inductivo Conceptual (1985)

Wood (1991a) Inductivo Conceptual

\footnotetext{
* Teoría no considerada por McWilliams et al. (2006) pero su naturaleza fue explicitada por los autores referidos.

** El relativismo ético no fue considerado por McWilliams et al. (2006) por lo que la naturaleza teórica fue propuesta por el autor de este trabajo al estar implícita en los trabajos aquí referidos.
}

Fuente: Elaboración propia.

\section{4) Teorías éticas}

Garriga y Melé la subdividieron también en cuatro clases: a) Teoría normativa de los grupos de interés (stakeholders), b) Derechos humanos, c) Desarrollo sostenible y d) Enfoque del bien común. El enfoque primario de esta categoría es hacia "los requerimientos éticos que cimentan las relaciones entre las empresas y la sociedad” (Garriga y Melé, 2004: 60). En este caso, de las 18 contribuciones clasificadas en el cuadro 5 , se observa concentración de investigadores de marketing en la subcategoría Desarrollo sostenible. 
Marketing y Responsabilidad

Social Empresarial

Cuadro 5. Categoría de las teorías éticas

\begin{tabular}{|c|c|c|c|}
\hline Naturaleza de la perspectiva teórica & $\begin{array}{l}\text { Contribuciones } \\
\text { clave }\end{array}$ & Método & $\begin{array}{l}\text { Tipo de } \\
\text { trabajo }\end{array}$ \\
\hline \multicolumn{4}{|c|}{ Subcategoría: Teoría normativa de los grupos de interés [stakeholders] } \\
\hline \multirow{3}{*}{$\begin{array}{l}\text { Ética aplicada a la empresa* / } \\
\text { Teoría de los grupos de interés }\end{array}$} & Carroll (199I) & Inductivo & Conceptual \\
\hline & $\begin{array}{l}\text { Freeman, Wicks y } \\
\text { Parmar (2004) }\end{array}$ & $\begin{array}{l}\text { Discurso } \\
\text { argumentativo }\end{array}$ & Conceptual \\
\hline & $\begin{array}{l}\text { Donaldson y } \\
\text { Davis (1991) }\end{array}$ & $\begin{array}{l}\text { Hipotético- } \\
\text { deductivo }\end{array}$ & Predictivo \\
\hline $\begin{array}{l}\text { Ética aplicada a la empresa* / Teoría } \\
\text { de los grupos de interés / Teoría de } \\
\text { la propiedad* }\end{array}$ & $\begin{array}{l}\text { Donaldson y } \\
\text { Preston (1995) }\end{array}$ & Inductivo & Conceptual \\
\hline $\begin{array}{l}\text { Ética aplicada a la empresa*/ } \\
\text { Democracia corporativa* / Teoría de } \\
\text { los grupos de interés }\end{array}$ & $\begin{array}{l}\text { Freeman y Reed } \\
\text { (1983) }\end{array}$ & Inductivo & Conceptual \\
\hline $\begin{array}{l}\text { Democracia corporativa* / Teoría de } \\
\text { los grupos de interés / Teoría integra- } \\
\text { dora del contrato social* }\end{array}$ & Freeman (1994) & Inductivo & Conceptual \\
\hline $\begin{array}{l}\text { Ética aplicada a la empresa* / Teoría } \\
\text { de los grupos de interés /Teoría } \\
\text { integradora del contrato social* }\end{array}$ & $\begin{array}{l}\text { Phillips, Freeman } \\
\text { y Wicks (2003) }\end{array}$ & $\begin{array}{l}\text { Discurso } \\
\text { argumentativo }\end{array}$ & Conceptual \\
\hline
\end{tabular}

Subcategoría: Derechos humanos

Ética aplicada a la empresa* / Teoría de Logsdon y Wood Inductivo Normativo los grupos de interés /Ciudadanía cor- (2002) porativa* /Ciudadanía de la empresa*

Nisión estratégica de la firma

Derechos humanos*

Derechos humanos*/Ciudadanía corporativa*
Wellford (2002) Inductivo

Conceptual

Wettstein y

Waddock (2005)
Inductivo

Explorato-

rio 
Cuadro 5. Categoría de las teorías éticas

(Continuación)

\begin{tabular}{|c|c|c|c|}
\hline Naturaleza de la perspectiva teórica & $\begin{array}{c}\text { Contribuciones } \\
\text { clave }\end{array}$ & Método & $\begin{array}{l}\text { Tipo de } \\
\text { trabajo }\end{array}$ \\
\hline \multicolumn{4}{|c|}{ Subcategoría: Desarrollo sostenible } \\
\hline \multirow[t]{3}{*}{$\begin{array}{l}\text { Teoría del desarrollo sostenible* } \\
\text { [Sustainable development] }\end{array}$} & $\begin{array}{l}\text { Bhaskaran, } \\
\text { Polonsky, Cary y } \\
\text { Fernandez (2006) }\end{array}$ & $\begin{array}{l}\text { Hipotético- } \\
\text { deductivo }\end{array}$ & Predictivo \\
\hline & $\begin{array}{l}\text { Gladwin, Kenelly y } \\
\text { Krause (1995) }\end{array}$ & Inductivo & Conceptual \\
\hline & $\begin{array}{l}\text { van Marrewijk } \\
(2003)\end{array}$ & Inductivo & Conceptual \\
\hline $\begin{array}{l}\text { Teoría del desarrollo sostenible* / } \\
\text { Teoría de los grupos de interés }\end{array}$ & $\begin{array}{l}\text { Byrne y Polonsky } \\
(2001)\end{array}$ & Inductivo & Conceptual \\
\hline \multirow[t]{2}{*}{$\begin{array}{l}\text { Teoría del desarrollo sostenible* } \\
\text { /Teoría institucional }\end{array}$} & $\begin{array}{l}\text { Jennings y Zand- } \\
\text { bergen (1995) }\end{array}$ & Inductivo & $\begin{array}{l}\text { Explorato- } \\
\text { rio }\end{array}$ \\
\hline & \multicolumn{3}{|c|}{ Subcategoría: Enfoque del bien común } \\
\hline $\begin{array}{l}\text { Teoría de los grupos de interés / } \\
\text { Teoría del bien común* }\end{array}$ & Argandoña (1998) & Inductivo & Conceptual \\
\hline $\begin{array}{l}\text { Teoría de la virtud* [Virtue theory] } \\
\text { /Teoría del bien común* /Visión estra- } \\
\text { tégica de la firma /Teoría del servidor }\end{array}$ & Arjoon (2000) & Inductivo & Conceptual \\
\hline \multirow[t]{2}{*}{$\begin{array}{l}\text { Teoría del bien común*/Teoría kyosei } \\
\text { [Kyosei theory]* }\end{array}$} & Kaku (1997) & $\begin{array}{l}\text { Estudio de } \\
\text { caso }\end{array}$ & Instrumental \\
\hline & $\begin{array}{l}\text { Wokutch y She- } \\
\text { pard (1999) }\end{array}$ & Inductivo & Conceptual \\
\hline
\end{tabular}

* Teoría no considerada por McWilliams et al. (2006) pero cuya naturaleza fue explicitada por los autores referidos en la tabla.

Fuente: Elaboración propia. 
Marketing y Responsabilidad

Social Empresarial

\section{Panorama general de las teorías usadas en la RSE}

Una vez identificadas y clasificadas las principales perspectivas teóricas que han sido usadas para el estudio de la RSE es posible obtener una panorámica general de su utilización de acuerdo con las catorce subcategorías antes descritas, ésta se presenta en el cuadro 6.

Cuadro 6. Panorama general de las PeRSPECtivas teóricas usadas PARA EL ESTUDIO DE LA RSE.

\begin{tabular}{|c|c|c|c|c|c|c|c|c|c|c|c|c|c|c|c|c|c|c|c|c|}
\hline $\begin{array}{l}\text { Teoría, principio } \\
\text { o visión de } \\
\text { Subcategoría }\end{array}$ & 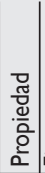 & $\sum_{\substack{=\\
亡}}$ & $\mid \begin{array}{l}. \frac{\pi}{U} \\
\frac{c}{0} \\
\frac{0}{\alpha} \\
\end{array}$ & 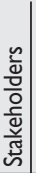 & 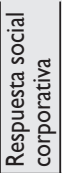 & 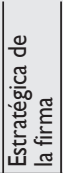 & 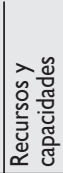 & 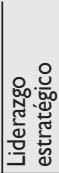 & 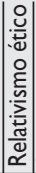 & 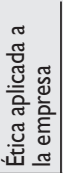 & 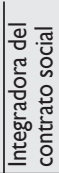 & 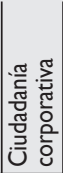 & 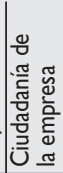 & $=$ & 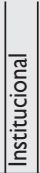 & 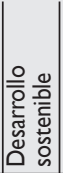 & 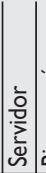 & 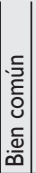 & 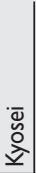 & \\
\hline $\begin{array}{l}\text { Maximización del valor } \\
\text { de los accionistas }\end{array}$ & & & $\bullet$ & & & & & & - & & & & & & & & & & & \\
\hline $\begin{array}{l}\text { Estrategias para lograr } \\
\text { ventajas competitivas }\end{array}$ & & $\bullet$ & & & & • & $\bullet$ & • & & & & & & & & & & & & \\
\hline Marketing con causa & & $\bullet$ & & & & $\bullet$ & & & & & & & & & & & & & & \\
\hline $\begin{array}{l}\text { Constitucionalismo } \\
\text { corporativo }\end{array}$ & & & & & & & & & & & & & & & & & • & & & \\
\hline $\begin{array}{l}\text { Integradoras del } \\
\text { contrato social }\end{array}$ & & & & & & & & & & & • & & & & & & & & & \\
\hline Ciudadanía corporativa & & & & & & & & & & & & - & & & & & & & & \\
\hline Aspectos de gestión & & $\bullet$ & & - & - & - & & & - & - & - & & & - & & & & & & \\
\hline $\begin{array}{l}\text { Principio de responsa- } \\
\text { bilidad pública }\end{array}$ & & & $\bullet$ & $\bullet$ & $\bullet$ & & • & & & & & & & • & & & & & & \\
\hline $\begin{array}{l}\text { Gestión de los } \\
\text { stakeholders }\end{array}$ & $\bullet$ & $\bullet$ & - & • & & - & • & & & •• & • & & & & & & - & & & \\
\hline $\begin{array}{l}\text { Desempeño social } \\
\text { corporativo }\end{array}$ & $\bullet$ & $\bullet$ & $\bullet$ & & $\bullet$ & • & & & $\bullet$ & $\bullet$ & & & & & & & $\bullet$ & & & \\
\hline $\begin{array}{l}\text { Teoría corporativa } \\
\text { de los stakeholders }\end{array}$ & • & & & - & & & & & & - & • & & & • & & & & & & \\
\hline Derechos humanos & & & & $\bullet$ & & & & & & - & - & & & - & & & & & & \\
\hline Desarrollo sostenible & & & & $\bullet$ & & - & & & & $\bullet$ & & - & - & & & & & & & \\
\hline Desarrollo sostenible & & & & $\bullet$ & & & & & & & & & & & $\bullet$ & $\bullet$ & & & & \\
\hline Enfoque del bien común & & & & $\bullet$ & & - & & & & & & & & & & & • & $\bullet$ & $\bullet$ & • \\
\hline
\end{tabular}

Fuente: Elaboración propia. 


\section{Marketing y RSE}

Si bien los cuadros anteriores arrojan información adecuada para responder al cuestionamiento sobre las principales perspectivas teóricas con las que ha sido estudiada la RSE y su naturaleza, para poder abordar la clasificación específica referente al marketing es necesario identificar primero los nexos conceptuales entre ambas esferas de conocimiento; sin embargo, debido a que dicha identificación excede claramente los alcances de este documento, éstos se exponen a continuación únicamente con carácter ilustrativo (para una discusión detallada sobre el tema puede verse Alvarado, 2007).Análisis de primero y segundo nivel, respectivamente, permite reconocer que existen al menos ocho nexos entre la definición más reciente de marketing y la RSE, a saber:a) creación, comunicación y entrega de valor a los consumidores; b) gestión de relaciones con el consumidor; c) beneficios mutuos; d) stakeholders; e) intercambio mixto; f) interactivo; g) interdependiente, en un entorno de h) marketing ampliado.

\section{La RSE en el Marketing}

Listados los principales nexos marketing-RSE es viable exponer las principales perspectivas con que la RSE ha sido abordada desde el marketing para responder a la segunda pregunta planteada. Para ello se recurrió al trabajo de investigación de Maignan y Ferrell, que si bien no fue desarrollado como una clasificación ex profeso, si identifica las tres perspectivas que mayoritariamente han examinado la RSE en la literatura de marketing "en términos de la unidad de análisis considerada y de las dimensiones de la responsabilidad social investigadas" (Maignan y Ferrell, 2004: 4), con lo cual permite la elaboración final del esquema de clasificación propuesto. Dichas perspectivas son: I) La relación entre las acciones de RSE y el desempeño financiero de la empresa, 2) La importancia atribuida por los gerentes y practicantes de marketing hacia la ética y a la responsabilidad social,y 3) La respuesta del consumidor ante las iniciativas de responsabilidad social de las empresas (Maignan y Ferrell, 2004); no obstante, en la presente investigación se ha considerado una categoría adicional que permite incorporar las investigaciones relativas a: 4) La integración teórica de la RSE en marketing. En el cuadro 7 se recogen las contribuciones más representativas señalando su objeto de estudio, su(s) autor(es) y el journal en el que fueron publicadas originalmente. 
Marketing y Responsabilidad

Social Empresarial

Las contribuciones en cuestión corresponden a los siguientes journals:

- AMAC American Marketing Association Conference Proceedings

- B\&S Business and Society

- BSR Business and Society Review

- CMR California Management Review

- EjM European Journal of Marketing

- JAMS Journal of the Academy of Marketing Science

- JBE Journal of Business Ethics

- JBR Journal of Business Research

- JCM Journal of Consumer Marketing

- JM Journal of Marketing

- JMR Journal of Marketing Research

- JPPM Journal of Public Policy \& Marketing

- JR Journal of Retailing

- tamr The Academy of Management Review

- UBR Universia Business Review

Cuadro 7. Contribuciones Representativas de los ASPECTOS DE RSE ESTUDIADOS EN MARKETING

\begin{tabular}{|c|c|c|c|}
\hline 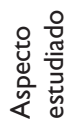 & Objeto de estudio & Autores & $\begin{array}{l}\overline{\bar{z}} \\
\text { ș } \\
\text { 으 }\end{array}$ \\
\hline \multirow{7}{*}{ 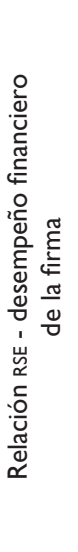 } & $\begin{array}{l}\text { Relación entre la respuesta a stakeholders clave y la } \\
\text { efectividad organizacional }\end{array}$ & $\begin{array}{l}\text { Koll,Woodside, y } \\
\text { Mühlbacher (2005) }\end{array}$ & EJM \\
\hline & Ciudadanía corporativa y beneficios económicos & Maignan, Ferrell y Hult (1999) & JAMS \\
\hline & $\begin{array}{l}\text { Marketing medioambiental como fuente de reputación } \\
\text { y su relación con el desempeño financiero de la firma }\end{array}$ & Miles y Covin (2000) & JBE \\
\hline & $\begin{array}{l}\text { Medición holística del desempeño del marketing a } \\
\text { partir de las percepciones de los stakeholders }\end{array}$ & $\begin{array}{l}\text { Murphy, Maguiness, Pescott, } \\
\text { Wislang, Ma y Wang (2005) }\end{array}$ & EJM \\
\hline & $\begin{array}{l}\text { Modelo sobre el rol de la reputación y las con- } \\
\text { tingencias de mercado y organizacionales en la } \\
\text { relación desempeño social corporativo-desempeño } \\
\text { financiero }\end{array}$ & $\begin{array}{l}\text { Neville, Bell y Mengüç } \\
(2005)\end{array}$ & EJM \\
\hline & Utilización de criterios de RSE entre inversionistas & Pava y Krausz (1996) & $\mathrm{JBE}$ \\
\hline & $\begin{array}{l}\text { Relación entre desempeño social corporativo y } \\
\text { desempeño financiero }\end{array}$ & Stanwick y Stanwick (1998) & JBE \\
\hline
\end{tabular}


Cuadro 7. Contribuciones Representativas de los ASPECtOS DE RSE

ESTUdiAdOS EN MARKETING

(Continuación)

\begin{tabular}{|c|c|c|c|}
\hline 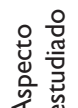 & Objeto de estudio & Autores & 혼 \\
\hline \multirow{7}{*}{ 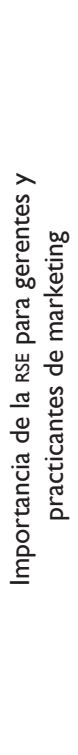 } & $\begin{array}{l}\text { Las alianzas sociales como medio para que los } \\
\text { empleados tenga una mayor identificación con la } \\
\text { empresa }\end{array}$ & $\begin{array}{l}\text { Berger, Cunningham, y } \\
\text { Drumwright (2006) }\end{array}$ & JAMS \\
\hline & $\begin{array}{l}\text { Grado de aplicación del ecologismo de la empresa } \\
\text { como estrategia de marketing en el sector de } \\
\text { bienes de consumo español y el compromiso de la } \\
\text { dirección en su adopción }\end{array}$ & Bravo, Fraj y Matute (2006) & UBR \\
\hline & $\begin{array}{l}\text { Preocupación medioambiental como criterio no } \\
\text { económico de compra organizacional }\end{array}$ & Drumwright (1994) & JM \\
\hline & $\begin{array}{l}\text { Modelo de análisis para la identificación de y } \\
\text { atención de las preocupaciones de los stakeholders } \\
\text { acerca de la publicidad de prescripción de medici- } \\
\text { nas directa al consumidor }\end{array}$ & Hoek y Maubach (2005) & EJM \\
\hline & $\begin{array}{l}\text { Influencia de las características corporativas e individua- } \\
\text { les en la orientación social de los gerentes }\end{array}$ & $\begin{array}{l}\text { Marz, Powers y Queisser } \\
(2003)\end{array}$ & JBE \\
\hline & $\begin{array}{l}\text { Desarrollo de una escala para medir la percepción de } \\
\text { los practicantes de marketing hacia la ética y la RSE }\end{array}$ & $\begin{array}{l}\text { Singhapakdi,Vitell, Rallapalli } \\
\text { y Kraft (1996) }\end{array}$ & JBE \\
\hline & $\begin{array}{l}\text { El rol de la ética normativa en el marketing y la } \\
\text { necesidad de desarrollar una teoría y directrices } \\
\text { éticas para el marketing }\end{array}$ & Smith (200I) & JBE \\
\hline \multirow{6}{*}{ 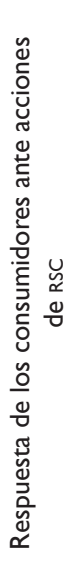 } & $\begin{array}{l}\text { Impacto del boicot de consumidores de países } \\
\text { poco desarrollados derivado de la irresponsabilidad } \\
\text { social corporativa de Nestlé }\end{array}$ & Baker (I 985) & $\mathrm{JBE}$ \\
\hline & $\begin{array}{l}\text { Influencia del marketing con causa en las decisiones } \\
\text { de compra del consumidor }\end{array}$ & $\begin{array}{l}\text { Barone, Miyazaki y Taylor } \\
(2000)\end{array}$ & JAMS \\
\hline & $\begin{array}{l}\text { Implementación y medición de las iniciativas de RSE } \\
\text { empleando factores resultantes internos y externos } \\
\text { del consumidor }\end{array}$ & Bhattacharya y Sen (2004) & CMR \\
\hline & $\begin{array}{l}\text { Relación entre RSE y estrategia de marca en la } \\
\text { respuesta del consumidor }\end{array}$ & $\begin{array}{l}\text { Berens, van Riel y van } \\
\text { Bruggen }(2005)\end{array}$ & JM \\
\hline & $\begin{array}{l}\text { Significado de la RSE entre consumidores de cuatro } \\
\text { países iberoamericanos }\end{array}$ & $\begin{array}{l}\text { Bigné, Chumpitaz, Andreu } \\
\text { y Swaen (2005) }\end{array}$ & UBR \\
\hline & $\begin{array}{l}\text { Asociaciones corporativas y respuesta de los } \\
\text { consumidores hacia compañías percibidas como } \\
\text { socialmente responsables }\end{array}$ & Brown y Dacin (1997) & JAM \\
\hline
\end{tabular}




\section{CuAdro 7. Contribuciones Representativas de LOS ASPECTOS DE RSE \\ ESTUDIADOS EN MARKETING \\ (Continuación)}

\begin{tabular}{|c|c|c|c|}
\hline 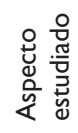 & Objeto de estudio & Autores & 홓 \\
\hline \multirow{12}{*}{ 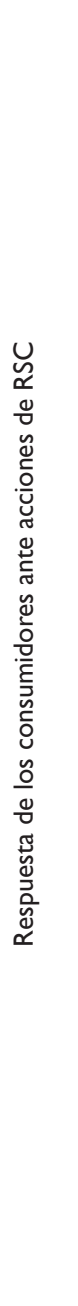 } & $\begin{array}{l}\text { Asociaciones e identidad corporativas, respuesta de } \\
\text { los consumidores hacia compañías percibidas como } \\
\text { socialmente responsables }\end{array}$ & Dacin y Brown (2006) & JAMS \\
\hline & $\begin{array}{l}\text { Influencia de las percepciones sobre aspectos } \\
\text { económicos, jurídicos, éticos y sociales en la evalua- } \\
\text { ción y lealtad de los consumidores de servicios de } \\
\text { telefonía móvil }\end{array}$ & $\begin{array}{l}\text { De los Salmones, Crespo y } \\
\text { Del Bosque (2005) }\end{array}$ & JBE \\
\hline & $\begin{array}{l}\text { Condiciones que contribuyen a una mejor evalua- } \\
\text { ción de los esfuerzos de marketing con causa }\end{array}$ & Ellen, Mohr y Webb (2000) & $\mathrm{JR}$ \\
\hline & $\begin{array}{l}\text { Atribuciones de los consumidores a los programas } \\
\text { de RSE }\end{array}$ & Ellen, Webb y Mohr (2006) & JAMS \\
\hline & $\begin{array}{l}\text { Disponibilidad a pagar un sobreprecio asociado a } \\
\text { un producto o servicio socialmente responsable }\end{array}$ & Fernández y Merino (2005) & UBR \\
\hline & $\begin{array}{l}\text { Variables psicológicas positivas (esperanza, gratitud, } \\
\text { espiritualidad y generosidad) como predictores de } \\
\text { la sensibilidad del consumidor hacia el desempeño } \\
\text { social corporativo }\end{array}$ & $\begin{array}{l}\text { Giacalone, Paul y } \\
\text { Jurkiewicz (2005) }\end{array}$ & JBE \\
\hline & $\begin{array}{l}\text { Influencia del marketing con causa en la construc- } \\
\text { ción de la equidad de marca }\end{array}$ & Hoeffler y Keller (2002) & JPPM \\
\hline & $\begin{array}{l}\text { Influencia de la credibilidad de los personajes que } \\
\text { respaldan las acciones de RSE sobre las actitudes de } \\
\text { los consumidores }\end{array}$ & $\begin{array}{l}\text { Lafferty y Goldsmith } \\
\text { (1999) }\end{array}$ & $\mathrm{JBR}$ \\
\hline & $\begin{array}{l}\text { Efecto de las alianzas marca-causa y la familiaridad } \\
\text { con la causa sobre las actitudes hacia la marca }\end{array}$ & $\begin{array}{l}\text { Lafferty y Goldsmith } \\
(2005)\end{array}$ & JBR \\
\hline & $\begin{array}{l}\text { Beneficios para las firmas y las ONG derivadas de } \\
\text { las percepciones de los consumidores sobre las } \\
\text { iniciativas de RSE: incremento en el comportamiento } \\
\text { real de compra y en la evaluación de las primeras e } \\
\text { incremento en las donaciones a las segundas }\end{array}$ & $\begin{array}{l}\text { Lichtenstein, Drumwright } \\
\text { y Braig (2004) }\end{array}$ & JM \\
\hline & $\begin{array}{l}\text { Relación entre RSE, satisfacción del consumidor y } \\
\text { valor de mercado de la firma }\end{array}$ & Luo y Bhattacharya (2006) & JM \\
\hline & $\begin{array}{l}\text { Sensibilidad de los consumidores de Europa occi- } \\
\text { dental hacia el desempeño social corporativo }\end{array}$ & Meijer y Schuyt (2005) & BYS \\
\hline
\end{tabular}


Cuadro 7. Contribuciones Representativas de los ASPECtOS DE RSE

ESTUdiAdOS EN MARKETING

(Continuación)

\begin{tabular}{|c|c|c|c|}
\hline 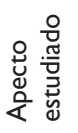 & Objeto de estudio & Autores & 홀 \\
\hline \multirow{6}{*}{ 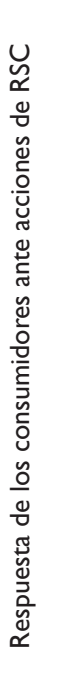 } & $\begin{array}{l}\text { Imagen del marketing como responsable de la } \\
\text { sociedad de consumo, su estilo de vida hedónico y } \\
\text { la erosión material de otras culturas y su relación } \\
\text { con la aceptación social }\end{array}$ & $\begin{array}{l}\text { O'Shaughnessy y Jackson } \\
\text { O'Shaughnessy (2002) }\end{array}$ & EMM \\
\hline & $\begin{array}{l}\text { La confianza en la fuente de marketing y las atri- } \\
\text { buciones del consumidor sobre la responsabilidad } \\
\text { como moderadores críticos en las estrategias de } \\
\text { posicionamiento socialmente responsables }\end{array}$ & Osterhus (1997) & JM \\
\hline & $\begin{array}{l}\text { Desarrollo de una escala de medición para medir la } \\
\text { sensibilidad del consumidor estadounidense hacia el } \\
\text { desempeño social corporativo }\end{array}$ & $\begin{array}{l}\text { Paul, Zalka, Downes, Perry } \\
\text { y Friday (1997) }\end{array}$ & BYS \\
\hline & $\begin{array}{l}\text { Efectos del género y la proximidad en las campañas } \\
\text { de marketing con causa }\end{array}$ & $\begin{array}{l}\text { Ross, Patterson y Stutts } \\
\text { (1992) }\end{array}$ & JAMS \\
\hline & $\begin{array}{l}\text { Efecto de las iniciativas de RSE en los juicios éticos } \\
\text { de los consumidores acerca de la empresa }\end{array}$ & Sen y Bhattacharya (200I) & JMR \\
\hline & $\begin{array}{l}\text { Grado de atención del consumidor a acciones de } \\
\text { RSE y su impacto en intenciones de comportamiento } \\
\text { futuro en cuanto a consumo, empleo e inversión }\end{array}$ & $\begin{array}{l}\text { Sen, Bhattacharya y } \\
\text { Korschun (2006) }\end{array}$ & JAMS \\
\hline \multirow{7}{*}{ 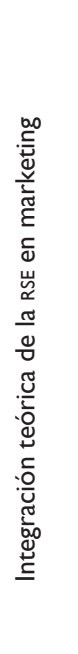 } & $\begin{array}{l}\text { Forma en que la identidad corporativa refleja las } \\
\text { afinidades de empleados y de la organización y } \\
\text { forma en la que es percibida por otros stakeholders }\end{array}$ & Balmer $(200 \mathrm{I})$ & EMM \\
\hline & $\begin{array}{l}\text { Integración de la teoría de los stakeholders, el } \\
\text { marketing relacional, la RSE y la creación de valor en } \\
\text { un contexto no occidental }\end{array}$ & Cai y Wheale (2004) & BSR \\
\hline & El rol de los stakeholders en la creación de valor & Clulow (2005) & EMM \\
\hline & $\begin{array}{l}\text { Reconceptualización de la satisfacción del consumi- } \\
\text { dor a partir de la RSE }\end{array}$ & Daub y Ergenzinger (2005) & EJM \\
\hline & $\begin{array}{l}\text { La teoría integradora del contrato social como } \\
\text { fundamento normativo para el pensamiento de } \\
\text { marketing }\end{array}$ & $\begin{array}{l}\text { Dunfee, Smith y Ross } \\
\text { (1999) }\end{array}$ & JM \\
\hline & $\begin{array}{l}\text { Nexos entre las prácticas de RSE, los nuevos riesgos } \\
\text { de gestión de marcas corporativas, el contexto orga- } \\
\text { nizacional y la construcción efectiva de una marca }\end{array}$ & Kay (2006) & EMM \\
\hline & $\begin{array}{l}\text { El rol del marketing como líder en las actividades } \\
\text { estratégicas de RSE }\end{array}$ & Lantos (200I) & JCM \\
\hline
\end{tabular}




\section{Cuadro 7. Contribuciones Representativas de los ASPECtOS DE RSE \\ estudiados en Marketing \\ (Continuación)}

\begin{tabular}{|c|c|c|c|}
\hline 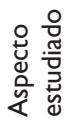 & Objeto de estudio & Autores & 竝 \\
\hline \multirow{13}{*}{ 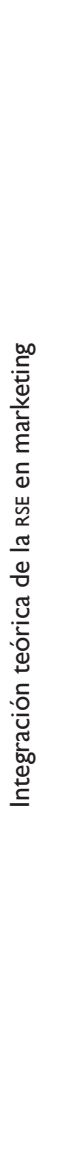 } & $\begin{array}{l}\text { Ciudadanía corporativa como herramienta de } \\
\text { marketing }\end{array}$ & Maignan y Ferrell (200I) & EJM \\
\hline & $\begin{array}{l}\text { Marco para integrar la RSE y el marketing basado en } \\
\text { la teoría de los stakeholders }\end{array}$ & Maignan y Ferrell (2004) & JAMS \\
\hline & $\begin{array}{l}\text { Modelo de implementación de la RSE basado en la } \\
\text { orientación a los stakeholders' }\end{array}$ & $\begin{array}{l}\text { Maignan, Ferrell y Ferrell } \\
(2005)\end{array}$ & EMM \\
\hline & $\begin{array}{l}\text { Ambientalismo corporativo como estrategia de } \\
\text { marketing }\end{array}$ & Menon y Menon (1997) & JM \\
\hline & $\begin{array}{l}\text { Uso de la teoría de los stakeholders para la cons- } \\
\text { trucción de marcas de eventos globales con fuertes } \\
\text { enlaces locales interorganizacionales }\end{array}$ & $\begin{array}{l}\text { Merrilees, Getz y O’Brien } \\
(2005)\end{array}$ & EJM \\
\hline & $\begin{array}{l}\text { Impactos de la certificación SA8000 en las activida- } \\
\text { des de marketing }\end{array}$ & Miles y Munilla (2004) & JBE \\
\hline & $\begin{array}{l}\text { Modelo integrador de la RSE y el marketing vía la } \\
\text { orientación al mercado }\end{array}$ & Murray y Montanari (1986) & TAMR \\
\hline & $\begin{array}{l}\text { La matriz estratégica de los stakeholders como guía } \\
\text { para tratar con los grupos de interés }\end{array}$ & Polonsky y Scott (2005) & EJM \\
\hline & $\begin{array}{l}\text { Identificación de los stakeholders en el contexto } \\
\text { detallista internacional }\end{array}$ & Quinn y Palmer (2005) & EJM \\
\hline & $\begin{array}{l}\text { Integración de la RSE y la ética en los procesos de } \\
\text { planeación estratégica de marketing }\end{array}$ & Robin y Reidenbach (1987) & JM \\
\hline & $\begin{array}{l}\text { Efectos de la reputación del comprador en la repu- } \\
\text { tación del vendedor en relación a los factores de RSE }\end{array}$ & Shi (2006) & AMAC \\
\hline & $\begin{array}{l}\text { El desarrollo de nuevos productos a partir de la } \\
\text { relevancia de los stakeholders }\end{array}$ & Smith y Fischbacher (2005) & EJM \\
\hline & $\begin{array}{l}\text { Necesidad de revitalizar los límites del marketing a } \\
\text { una perspectiva que permita incorporar los aspec- } \\
\text { tos económicos, ecológicos y sociales mediante la } \\
\text { circulación total de los componentes e interfases } \\
\text { de los canales de marketing }\end{array}$ & Svensson (2005) & EMM \\
\hline
\end{tabular}

'De acuerdo con los autores, el constructo de orientación a los stakeholders permite una mejor integración RSE-marketing que el de orientación al mercado pues éste último suele enfocarse en un sólo grupo de interés: los consumidores (Maignan, Ferrell y Ferrell, 2005).

${ }^{2}$ Svensson acuñó el término Concepto Esférico de Marketing para subsanar dicha necesidad (2005). 
Cuadro 7. Contribuciones Representativas de los ASPECtOS DE RSE

ESTUdiAdos en MARKETING

(Continuación)

\begin{tabular}{|c|c|c|c|}
\hline 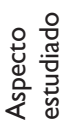 & Objeto de estudio & Autores & 홍 \\
\hline \multirow{6}{*}{ 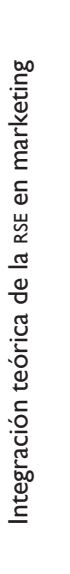 } & $\begin{array}{l}\text { Fijación de precios socialmente responsables en la } \\
\text { industria farmacéutica }\end{array}$ & Vachani y Smith (2004) & CMR \\
\hline & $\begin{array}{l}\text { Exploración de las relaciones entre los comporta- } \\
\text { mientos empresariales y el apoyo a stakeholders } \\
\text { con intereses divergentes }\end{array}$ & $\begin{array}{l}\text { Voss, Voss y Moorman } \\
(2005)\end{array}$ & EJM \\
\hline & Conceptualización del marketing con causa & $\begin{array}{l}\text { Varadarajan y Menon } \\
\text { (1988) }\end{array}$ & JM \\
\hline & $\begin{array}{l}\text { Los comunicados de prensa como indicadores de } \\
\text { compromiso con los stakeholders }\end{array}$ & Whysall (2005) & EJM \\
\hline & $\begin{array}{l}\text { Interfaces gerenciales para la identificación de los } \\
\text { stakeholders directos e indirectos en la actividad } \\
\text { de servicios }\end{array}$ & Wood y Jallat (2005) & EJM \\
\hline & $\begin{array}{l}\text { Forma en que los observadores emplean las asocia- } \\
\text { ciones corporativas }{ }^{3} \text { para inferir la disposición de } \\
\text { los consumidores }\end{array}$ & $\begin{array}{l}\text { Yoon, Gurhan-Canli y } \\
\text { Bozok (2006) }\end{array}$ & JAMS \\
\hline
\end{tabular}

${ }^{3}$ Corporate Associations.

Fuente: Elaboración propia.

\section{Conclusiones}

Las clasificaciones propuestas y empleadas en los epígrafes precedentes no pueden -ni pretenden- ser cámaras estancas concluyentes, sino representaciones que contribuyan a la mejor comprensión de la estructura de las investigaciones sobre RSE en general y en el marketing en particular. Esto se debe a diferentes razones: en primer término, a que la poca claridad fronteriza que caracteriza a diversos aspectos del marketing $-\mathrm{y}$ de la propia RSE- ocasiona que sus categorizaciones suelan ser arbitrarias en alguna medida (Gummesson, 1987) aun cuando éstas son valiosos vehículos de pensamiento (Gummesson, 1987) y análisis (Hunt, 1976); además, las particularidades de las bases de datos empleadas para la obtención de los artículos estudiados introducen un sesgo derivado del 
predominio de documentos en lengua inglesa y también a que, dadas la gran cantidad de investigaciones sobre RSE y las limitaciones de espacio, resulta imposible incorporar todas y cada una de las contribuciones en cuestión a este mapa, por lo que solamente contiene algunas de aquellas que han sido reconocidas como representativas, claves o seminales en la propia literatura y que proceden de journals líderes en sus respectivas esferas de conocimiento. Lo anterior sugiere la necesidad de llevar a cabo estudios adicionales de carácter cuantitativo que permitan la incorporación de un mayor número de contribuciones a través de análisis bibliométricos o de contenido.

Pese a las limitaciones señaladas, esta exploración de los últimos treinta y cinco años de literatura sobre RSE responde a lagunas concretas en el conocimiento de ésta en el marketing. En primera instancia, se integraron tres clasificaciones que permitieron considerar simultáneamente su naturaleza teórica (McWilliams et al., 2006), su enfoque en función del rol de los negocios en la sociedad (Garriga y Melé, 2004) y el tipo de trabajo intelectual y los métodos empleados para la generación de sus contenidos (De Bakker et al., 2005 y 2006), lo que hizo posible a su vez la construcción de una panorámica general de los estudios de RSE en el management y el marketing. En segundo lugar, el análisis del proceso de redefinición del marketing ha favorecido la identificación y el establecimiento de los vínculos conceptuales existentes entre el paradigma emergente del marketing y la RSE y, en tercer término, la identificación de las principales perspectivas con las que los especialistas en marketing han estudiado la RSE ha servido para proponer, elaborar y aplicar una clasificación a sus trabajos.

En su conjunto, esta aportación resulta útil tanto para el trabajo académico como para la práctica del marketing, pues si hemos de recorrer los largos caminos referidos, es prudente contar con un mapa de navegación flexible y adecuado, y reducir, en lo posible, la confusión sobre el tema.

\section{FUENTES CONSULTADAS}

Ackerman, Robert W. (1973). "How companies respond to social demands", Harvard Business Review, 5 I (4), 88.

Agle, Bradley R., Ronald K. Mitchell y Jeffrey A. Sonnenfeld (1999). "Who matters to CEOs? An investigation of stakeholder attributes and salience, 
corporate performance, and CEO values". Academy of Management Journal, 42(5), 507-525.

Alvarado Herrera,Alejandro (2007). Evolución epistemológica de la responsabilidad social empresarial: del management al marketing. Valencia: Universitat de Valencia.

Argandoña,Antonio (1998). "The stakeholder theory and the common good”. Journal of Business Ethics, 17(9), 1093-1 102.

Arjoon, Surendra (2000). "Virtue theory as a dynamic theory of business". Journal of Business Ethics, 28(2), 159.

Asher, Cheryl Carleton, James M. Mahoney y Joseph T. Mahoney (2005). “Towards a property rights foundation for a stakeholder theory of the firm". Journal of Management y Governance, 9(I), 5.

Baker, James C. (1985). "The international infant formula controversy: a dilemma in corporate social responsibility". Journal of Business Ethics, $4(3), 181$.

Balmer,John M.T.(200I).“Corporate identity, corporate branding and corporate marketing - Seeing through the fog”, European Journal of Marketing, 35(3/4), 248.

Ballesteros, Carlos (2004). “Editorial”. Investigación y Marketing, (85), 4.

Baron, David P. (200I). "Private politics, corporate social responsibility and integrated strategy”, Journal of Economics y Management Strategy, I0(I), 7-45.

Barone, Michael J.,Anthony D. Miyazaki y Kimberly A.Taylor (2000).“The influence of cause-related marketing on consumer choice: does one good turn deserve another?". Journal of the Academy of Marketing Science, 28(2), 248-262.

Berens, Guido,Van Riel, B. M. Cees y Gerrit H.Van Bruggen (2005). “Corporate associations and consumer product responses: the moderating role of corporate brand dominance", Journal of Marketing, 69(3), 35-48.

Berger, I. E., Cunningham, P. H. y M. E. Drumwright (2006). "Identity, identification, and relationship through social alliances", Journal of the Academy of Marketing Science, 34(2), I28- 37.

Bhaskaran, Suku, Michael Polonsky, John Cary y Shadwell Fernández (2006). "Environmentally sustainable food production and marketing", British Food Journal, I08(8), 677.

Bhattacharya, C. B y Sankar Sen (2004). “Doing better at doing good: when, 
why, and how consumers respond to corporate social initiatives". California Management Review, 47(I), 9.

Bhide,Amar y Howard H. Stevenson (1990). "Why be honest if honesty doesn't pay?". Harvard Business Review, 68(I2I).

Bigné, Enrique, Rubén Chumpitaz, Luisa Andreu y Valerie Swaen (2005). “Percepción de la responsabilidad social corporativa: un análisis crosscultural". Universia Business Review, 5, 14-27.

Blowfield, Michael (2004). "Implementation deficits of ethical trade systems: lessons from the Indonesian cocoa and timber industries". The Journal of Corporate Citizenship, (13), 77.

Bowen, Howard R. (1953). Social responsibilities of the businessman. Nueva York: Harper y Row.

Bravo Gil, Rafael, Elena Fraj Andrés y Jorge MatuteVallejo, (2006).“'La estrategia de 'ecologismo de empresas' en el sector de bienes de consumo final”. Universia Business Review, (9), 58-73.

Brown, Tom J. y Peter A. Dacin (1997). "The company and the product: corporate associations and consumer product responses". Journal of Marketing, $6 \mathrm{I}(\mathrm{I}), 68-84$.

Byrne, Michael Raymond, y Michael Jay Polonsky (200I). "Impediments to consumer adoption of sustainable transportation: alternative fuel vehicles". International Journal of Operations \& Production Management, $2 I(I 2), I 52 I$.

Cai, Zhuang, y Peter Wheale (2004). "Creating sustainable corporate value: a case study of stakeholder relationship management in China". Business and Society Review, I09(4), 507.

Caldwell, Cam y Ranjan Karri (2005). "Organizational governance and ethical systems: a covenantal approach to building trust".Journal of Business Ethics, 58(I-3), 249.

Carr, Albert (1968). "Is business bluffing ethical?". Harvard Business Review, 46(I), 143.

Carrigan, Marylyn y Ahmad Attalla (200I). "The myth of the ethical consumer - do ethics matter in purchase behaviour?". Journal of Consumer Marketing, 560-578.

Carroll,Archie B. ( 1979). "A three-dimensional conceptual model of corporate performance". The Academy of Management Review, 4(4), 17. 
(I99I). "The pyramid of corporate social responsibility: toward the moral management of organizational stakeholders". Business Horizons, 34(4), 39.

(1999). "Corporate social responsibility: evolution of a definitional construct”. Business and Society, 38(3), 268-295.

Clulow, Val (2005). "Futures dilemmas for marketers: can stakeholder analysis add value?". European Journal of Marketing, 39(9//0), 978- 997.

Coelho, Philip R. P. (I99I). "The pyramid of corporate social responsibility: toward the moral management of organizational stakeholders". Business Horizons, 34(4), 39. (2003b). "The social responsibility of management: a reprise". Mid-American Journal of Business, I8(2), 5 I.

James E. McClure y John A. Spry (2003a). "The social responsibility of corporate management: a classical critique". Mid-American Journal of Business, I8(I), I5.

Dacin, Peter A. y, Tom J. Brown (2006). “Corporate branding, identity, and customer response". Journal of the Academy of Marketing Science, 34(2), 95-98.

Daub, Claus-Heinrich y Rudolf Ergenzinger (2005). "Enabling sustainable management through a new multi-disciplinary concept of customer satisfaction”. European Journal of Marketing, 39(9/I0), 998-I0I 2.

Davis, K.(1967)."Understanding the social responsibility puzzle: what does the businessman owe to society?". Business Horizons, I0, 45.

De Bakker, Frank G. A., Peter Groenewegen y Frank Den Hond (2005). “A bibliometric analysis of 30 years of research and theory on corporate social responsibility and corporate social performance". Business and Society, 44(3), 283-317.

Groenewegen, Peter y Frank Den Hond (2006). “A research note on the use of bibliometrics to review the corporate social responsibility and corporate social performance literature". Business and Society, 45(I), 7-I9.

De los Salmones, M.D.G.,A. H. Crespo e I. R. del Bosque (2005). "Influence of corporate social responsibility on loyalty and valuation of services". Journal of Business Ethics, 6I (4), 369-385.

Donaldson, Lex y James H. Davis (I99I). "Stewardship theory or agency theory: ceo governance and shareholder returns". Australian Journal of Management, I6(I), 49. 
Donaldson, Thomas y Thomas W. Dunfee (1994).“Toward a unified conception of business ethics: integrative social contracts theory". The Academy of Management Review, I9(2), 252.

— y Thomas W. Dunfee (1999)."When ethics travel: the promise and peril of global business ethics". California Management Review, 4 I (4), 45.

- y Lee E. Preston (1995). "The stakeholder theory of the corporation: concepts, evidence, and implications". The Academy of Management Review, 20(I), 65-9I.

Drumwright, Minette E. (1994). "Socially responsible organizational buying: environmental concern as a noneconomic buying criterion”. Journal of Marketing, 58(3), I-I9.

Dunfee, Thomas W., N. Craig Smith y William T.Jr. Ross (1999)."Social contracts and marketing ethics". Journal of Marketing, 63(3), 14.

Ellen, Pam Scholder, Lois A. Mohr y Deborah J. Webb (2000). "Charitable programs and the retailer: do they mix?". Journal of Retailing, 76(3), 393-406.

_ Deborah J.Webb y Lois A. Mohr (2006). "Building corporate associations: consumer attributions for corporate socially responsible programs”. Journal of the Academy of Marketing Science, 34(2), I47-I 57.

Epstein, Edwin M. (1987). "The corporate social-policy process - beyond business ethics, corporate social-responsibility, and corporate social responsiveness". California Management Review, 29(3), 99.

Feddersen,Timothy J.y Thomas W. Gilligan (200I)."Saints and markets:activists and the supply of credence goods".Journal of Economics y Management Strategy, I0(I), I49-I7I.

Fernández, Francisco (2004). “RSC: Un largo camino por recorrer”. Investigación y Marketing, (85), 36-38.

Fernández Kranz, Daniel y Anna Merino Castelló (2005). “¿Existe disponibilidad a pagar por responsabilidad social corporativa? Percepción de los consumidores". Universia Business Review, (7), 38-53.

Fieser, James (1996). "Do businesses have moral obligations beyond what the law requires?". Journal of Business Ethics, VI5(4), 457-468.

Fombrun, Charles J., Naomi A. Gardberg y Michael L. Barnett (2000). “Opportunity platforms and safety nets: corporate citizenship and reputational risk". Business and Society Review, I05(I), 85. 
Freeman, R. Edward (1994). "The politics of stakeholder theory: Some future directions”. Business Ethics Quarterly, 4(4), 409.

,Andrew C.Wicks y Bidhan Parmar (2004)." Stakeholder theory and "the corporate objective revisited”, Organization Science, I5(3), 364-369.

y David L. Reed (1983). "Stockholders and stakeholders: a new perspective on corporate governance". California Management Review, 25(3), 88.

Friedman, Milton (1962). Capitalism and freedom. Chicago: University of Chicago Press.

(1970). "The social responsibility of business is to increase its profits". The New York Times Magazine.

(1978). “The Friedman Credo”, Across the Board, I5(5), 82.

Garriga, Elisabet y Doménec Melé (2004). "Corporate social responsibility theories: mapping the territory". Journal of Business Ethics, 53(I-2), 5I-7I.

Gerde, Virginia W. y Richard E.Wokutch (1998). "25 years and going strong”. Business and Society, 37(4), $4 \mid 4$.

Giacalone, Robert A., Karen Paul y Carole L. Jurkiewicz (2005).“A preliminary investigation into the role of positive psychology in consumer sensitivity to corporate social performance". Journal of Business Ethics, 58(4), 295.

Gladwin,Thomas N., James J. Kennelly y Tara-Shelomith Krause (1995). “Shifting paradigms for sustainable development: implications for management theory and research". The Academy of Management Review, 20(4), 874-907.

Gummesson, Evert (1987).“The new marketing-developing long-term interactive relationships”. Long Range Planning, 20(4), 10.

Hart, Stuart L. (1995). “A natural-resource-based view of the firm”. The Academy of Management Review, 20(4), 986.

— y Christensen, Clayton M.(2002). "The great leap: driving innovation from the base of the pyramid". MIT Sloan Management Review, 44(I), $5 \mathrm{I}$.

Hoeffler, Steve y Kevin Lane Keller (2002). "Building brand equity through corporate societal marketing”. Journal of Public Policy y Marketing, $2 \mathrm{I}(\mathrm{I}), 78$. 
Hoek, Janet y Ninya Maubach (2005). “A model for addressing stakeholders' concerns about direct-to-consumer advertising of prescription medicines”. European Journal of Marketing, 39(9/I0), I I5 I - I I 65.

Hunt, Shelby D. (1976). “The nature and scope of marketing”.Journal of Marketing, 40(3), 17-28.

Jennings, P. Devereaux y Paul A. Zandbergen (1995). "Ecologically sustainable organizations:an institutional approach". The Academy of Management Review, 20(4), I0I5.

Jensen, M.C. (2000)."Value maximization, stakeholder theory, and the corporate objective function", en M. Beer y N. Nohria (eds.) (2000). Breaking the code of change. Boston: Harvard Business School Press.

Jones, Thomas M. (1980). “Corporate social responsibility revisited, redefined”. California Management Review, 22(3), 59.

(1995). "Instrumental stakeholder theory: a synthesis of ethics and". The Academy of Management Review, 20(2), 404.

Kaku, Ryuzaburo (1997). “The path of kyosei”. Harvard Business Review, 75(4), 55.

Kay, Mark J. (2006). "Strong brands and corporate brands". European Journal of Marketing, 40(7/8), 742.

Kochan, Thomas A. y Saul A. Rubinstein (2000). Toward a stakeholder theory of the firm: the saturn partnership". Organization Science, I I (4), 367 386.

Koll, Oliver, Arch G. Woodside y Hans Mühlbacher (2005). "Balanced versus focused responsiveness to core constituencies and organizational effectiveness". European Journal of Marketing, 39(9/10), I I66- II83.

Lafferty, Barbara A.y Ronald E. Goldsmith (1999). "Corporate credibility's role in consumers' attitudes and purchase intentions when a high versus a low credibility endorser is used in the ad". Journal of Business Research, 44(2), I09-I I6.

— - r Ronald E. Goldsmith (2005). "Cause-brand alliances: does the cause help the brand or does the brand help the cause?".Journal of Business Research, 58(4), 423-429.

Lantos, Geoffrey P. (200I). "The boundaries of strategic corporate social responsibility”. Journal of Consumer Marketing, I8(7), 595-632.

Lea, David (1999). “Corporate and public responsibility, stakeholder theory and the developing world". Business Ethics a European Review, 8(3), I5I. 
Lichtenstein, Donald R., Minette E. Drumwright y Bridgette M. Braig (2004).“The effect of corporate social responsibility on customer donations to corporate-supported nonprofits". Journal of Marketing, 68(4), 16-32.

Litz, Reginald A. (1996). "A resource-based-view of the socially responsible firm: stakeholder interdependence, ethical awareness, and issue responsiveness as strategic assets". Journal of Business Ethics, I5(I2), I355-I363.

Lockett,Andy, Jeremy Moon y Wayne Visser (2006)."Corporate social responsibility in management research: focus, nature, salience and sources of influence". Journal of Management Studies, 43(I), I I 5-I 36.

Logsdon, Jeanne M.y Donna J.Wood (2002)."Business citizenship: from domestic to global level of analysis". Business Ethics Quarterly, I2(2), I 55.

Luo, Xueming y C. B. Bhattacharya (2006). "Corporate social responsibility, customer satisfaction, and market value". Journal of Marketing, 70(4), I-I8.

Maignan, Isabelle, O.C. Ferrell y G.Tomas M. Hult (1999).“Corporate citizenship: cultural antecedents and business benefits". Journal of the Academy of Marketing Science, 27(4), 455-469.

- y O. C. Ferrell (2000). "Measuring corporate citizenship in two countries: The case of the United States and France". Journal of Business Ethics, 23(3), 283.

y O.C. Ferrell (200I)."Corporate citizenship as a marketing instrument - Concepts, evidence and research directions". European Journal of Marketing, 35(3/4), 457.

- y O. C. Ferrell (2004). "Corporate social responsibility and marketing: an integrative framework".Journal of the Academy of Marketing Science, 32(I), 3-19.

O.C. Ferrell y Linda Ferrell (2005).“A stakeholder model for implementing social responsibility in marketing”. European Journal of Marketing, 39(9/10), 956- 977.

_ y David A. Ralston (2002). “Corporate social responsibility in Europe and the U.S.: insights from businesses' self-presentations”. Journal of International Business Studies, 33(3), 497-5 I 4.

Malan, Daniel (2005). "Corporate citizens, colonialists, tourists or activists? Ethical challenges facing South African corporations in Africa". The Journal of Corporate Citizenship, ( I8), 49. 
Marín,Ainhoa (2004). “Del marketing con causa a la responsabilidad social de la empresa". Investigación y Marketing, (85), 48-5I.

Marz, J.W, T.L. Powers y T. Queisser (2003). “Corporate and individual influences on managers' social orientation”. Journal of Business Ethics, 46(I), I-I I.

McWilliams, Abagail, Donald S. Siegel y Patrick M.Wright (2006). "Corporate social responsibility: strategic implications (editorial)". Journal of Management Studies, 43(I), I- I8.

— David Van Fleet y Kenneth D. Cory (2002)."Raising rivals' costs through political strategy: an extension of resource-based theory". Journal of Management Studies, 39, 707-724.

— y Donald S. Siegel (200I). "Corporate social responsibility: a theory of the firm perspective". The Academy of Management Review, 26(I), I I 7.

Meijer, May-may y Theo Schuyt (2005). "Corporate social performance as a bottom line for consumers". Business and Society, 44(4), 442.

Menon, Anil y Ajay Menon (1997). "Enviropreneurial marketing strategy: The emergence of corporate environmentalism as market strategy". Journal of Marketing, 6I (I), $5 \mathrm{I}$.

Merrilees, Bill, Don Getz y Danny O'brien, (2005). “Marketing stakeholder analysis: branding the brisbane goodwill games". European Journal of Marketing, 39(9/10), 1060- 1077.

Miles, Morgan P.y Jeffrey G. Covin (2000). “Environmental marketing: a source of reputational, competitive, and financial advantage”. Journal of Business Ethics, 23(3), 299.

— y Linda S. Munilla (2004). "The potential impact of social accountability certification on marketing: a short note”. Journal of Business Ethics, 50(I), I.

Mitchell, Ronald K., Bradley R. Agle y Donna J.Wood (1997)."Toward a theory of stakeholder identification and salience: defining the principle of who and what really counts". The Academy of Management Review, 22(4), 853.

Moir, Lance (200I). "What do we mean by corporate social responsibility?". Corporate Governance, I (2), I6.

Murphy, Brian, Paul Maguiness, Chris Maguiness, Soren Wislang, Jingwu Ma y Rongmei Wang (2005). "Stakeholder perceptions presage holistic stakeholder relationship marketing performance". European Journal of Marketing, 39(9/I0), I049- 1059. 
Murphy, Patrick E. (1978). "An evolution: corporate social responsiveness". University of Michigan Business Review, 30(6), 19.

Murray, Keith B. y John R. Montanari (1986). "Strategic management of the socially responsible firm: integrating management and marketing theory". The Academy of Management Review, I I (4), 8I 5.

Nasi, Juha, Salme Nasi, Nelson Phillips y Stelios Zyglidopoulos (1997). "The evolution of corporate social responsiveness". Business and Society, 36(3), 296.

Neville, Benjamin A., Simon J. Bell y Bülent Mengüç (2005). “Corporate reputation, stakeholders and the social performance-financial performance relationship”. European Journal of Marketing, 39(9//0), I I84- II 98.

O'Shaughnessy, John y Nicholas Jackson O'Shaughnessy (2002). "Marketing, the consumer society and hedonism". European Journal of Marketing, 36(5/6), 524- 547.

Osterhus, Thomas L. (1997). "Pro-social consumer influence strategies: when and how do they work?". Journal of Marketing, 6I (4), I6-29.

Paul, Karen, Lori M. Zalka, Meredith Downes, Susan Perry y Shawnta Friday (1997). “U.S. consumer sensitivity to corporate social performance”. Business and Society, 36(4), 408.

Pava, Moses L. y Joshua Krausz (1996). "The association between corporate social-responsibility and financial performance: the paradox of social cost". Journal of Business Ethics, I5(3), 32I-357.

Phillips, Robert, R. Edward Freeman y Andrew C.Wicks (2003).'What stakeholder theory is not". Business Ethics Quarterly, I3(4), 479-502.

Polonsky, Michael Jay (2005). "Stakeholder thinking in marketing". European Journal of Marketing, 39(9//0), 953-955.

— y Don Scott (2005). “An empirical examination of the stakeholder strategy matrix”. European Journal of Marketing, 39(9/10), II99- 1215.

Porter, Michael E. y Kramer, Mark R. (2002). "The competitive advantage of corporate philanthropy". Harvard Business Review, 80(I2), 56.

Prahalad, C. K. y A. Hammond (2002). "Serving the world's poor, profitably". Harvard Business Review, 80(9), 48-59.

Preston, Lee E.y J. E. Post (1975). Private management and public policy.The principle of public responsibility. Englewood Cliffs, N.J.: Prentice Hall. 
Quinn, Barry y Mark Palmer (2005). "Stakeholder relationships in an international retailing context: an investment bank perspective". European Journal of Marketing, 39(9/10), I096-III 7.

Robin, Donald P.y R. Eric Reidenbach (1987). "Social responsibility, ethics, and marketing strategy: closing the gap between concept and application". Journal of Marketing, $5 \mathrm{I}$ (I), 44.

Ross, John K. III, Larry T. Patterson y Mary Ann Stutts (1992). "Consumer perceptions of organizations that use cause-related marketing”. Journal of the Academy of Marketing Science, 20(I), 93-97.

Rowley, Timothy J. (1997). "Moving beyond dyadic ties: a network theory of stakeholder influences". The Academy of Management Review, 22(4), 887-910.

Sen, Sankar, C. B. Bhattacharya y Daniel Korschun (2006). "The role of corporate social responsibility in strengthening multiple stakeholder relationships: a field experiment".Journal of the Academy of Marketing Science, 34(2), I58-166.

— y C. B. Bhattacharya (200I). "Does doing good always lead to doing better? Consumer reactions to corporate social responsibility". Journal of Marketing Research, 38(2), 225-243.

Sethi, S. Prakash (1975)."Dimensions of corporate social performance-an analytical framework”. California Management Review, I7(3), 58.

Shi,Tiebing (2006). "The effect of the buyer's reputation on the seller's reputation: a network perspective". American Marketing Association. Conference Proceedings, 17, 28.

Singhapakdi, Anusorn, Scott J. Vitell, Kumar C. Rallapalli y Kenneth L. Kraft (1996). "The perceived role of ethics and social responsibility: a scale development”. Journal of Business Ethics, I 5( I I), I I 3 I - I I 40.

Smith,Anne M.y Moira Fischbacher (2005).“New service development:a stakeholder perspective”. European Journal of Marketing, 39(9/I0), I025- 1048.

Smith, Craig N. (200I). "Ethical guidelines for marketing practice: a reply to gaski and some observations on the role of normative marketing ethics". Journal of Business Ethics, 32(I), 3-18.

Stanwick, Peter A.y Sarah D. Stanwick (1998)."The relationship between corporate social performance and organizational size, financial performance and environmental performance: an empirical examination”. Journal of Business Ethics, I7(2), 195-204. 
Svensson, Göran (2005). “The spherical marketing concept: a revitalization of the marketing concept". European Journal of Marketing, 39(I/2), 5.

Swanson, Diane L. (1995). "Addressing a theoretical problem by reorienting the corporate social performance model”. The Academy of Management Review, 20(I), 43.

(1999). "Toward an integrative theory of business and society: a research strategy for corporate social performance". The Academy of Management Review, 24(3), 506-521.

Vachani, Sushil y N. Craig Smith (2004). "Socially responsible pricing: lessons from the pricing of AIDS drugs in developing countries". California Management Review, 47(I), II 7.

Van Marrewijk, Marcel (2003). "Concepts and definitions of CSR and corporate sustainability: between agency and communion". Journal of Business Ethics, 44(2/3), 95.

Van Oosterhout, J.y Pursey P.M.A. R. Heugens (2006)."Much to do about nothing: a conceptual critique of CSR”. [en línea] ERIM Report Series Reference (August 14, 2006). Disponible en: http://ssrn.com/abstract=924505 Varadarajan, P. Rajan y Anil Menon (1988).“Cause-related marketing: a coalignment of marketing strategy and corporate philanthropy". Journal of Marketing, 52(3), 58.

Voss, Zannie Giraud, Glenn B.Voss y Christine Moorman (2005).“An empirical examination of the complex relationships between entrepreneurial orientation and stakeholder support". European Journal of Marketing, 39(9/10), II32- II50.

Waldman, David A., Donald S. Siegel y Mansour Javidan (2006). "Components of CEO transformational leadership and corporate social responsibility". Journal of Management Studies, in press.

Wartick, Steven L.y Philip L. Cochran (1985). "The evolution of the corporate social performance model". The Academy of Management Review, 10(4), 758-769.

- y John F. Mahon (1994)."Toward a substantive definition of the corporate issue construct: a review and synthesis of the literature". Business and Society, 33(3), 293.

Watson, George W., Jon M. Shepard y Carroll U. Stephens (I999). "Fairness and ideology". Business and Society, 38(I), 83. 
Webb, Deborah J.y Lois A. Mohr (1998). "A typology of consumer responses to cause-related marketing: from skeptics to socially concerned". Journal of Public Policy y Marketing, I7(2), 226-238.

Wettstein, Florian y Sandra Waddock (2005). "Voluntary or mandatory: that is (not) the question: linking corporate citizenship to human rights obligations for business". Zeitschrift für Wirtschafts-und Unternehmensethik, 6(3), 304.

Whysall, Paul (2005). "Retailers' press release activity: market signals for stakeholder engagement?". European Journal of Marketing, 39(9/10), III8-II3I.

Windsor, Duane (2006).“Corporate social responsibility: three key approaches”. Journal of Management Studies, 43(I), 93-II4.

Wokutch, Richard E. y Jon M. Shepard (1999). "The maturing of the Japanese economy: corporate social responsibility implications”. Business Ethics Quarterly, 9(3), 527.

Wood, Donna J.(1991a)."Corporate social performance revisited". The Academy of Management Review, 16(4), 691-718.

(199/b)."Social issues in management: theory and research in corporate social performance”. Journal of Management, I7(2), 383.

- y Jeanne M. Logsdon (2002)."Business citizenship: from individuals to organizations". Business Ethics Quarterly, Ruffin Series (3), 59.

Wood, Elliot y Frédéric Jallat (2005).“Exploring “deep” and “wide” stakeholder relations in service activity". European Journal of Marketing, 39(9//0), I0I3-1024.

Yoon, Y., Z. Gurhan-Canli y B. Bozok (2006). "Drawing inferences about others on the basis of corporate associations". Journal of the Academy of Marketing Science, 34(2), I67-I 73.

Zenisek, Thomas J. (1979) "Corporate social responsibility: a conceptualization based on organizational literature". The Academy of Management Review, 1979, 4(3), 359. 